SMI-09-98

\title{
On the Breaking of Conformal Symmetry in the AdS/CFT Correspondence
}

\author{
I.Ya. Aref'eva and I.V. Volovich \\ Steklov Mathematical Institute, Russian Academy of Sciences \\ Gubkin St.8, GSP-1, 117966, Moscow, Russia \\ arefeva, volovich@mi.ras.ru
}

\begin{abstract}
The renormalization of the boundary action in the AdS/CFT correspondence is considered and the breaking of conformal symmetry is discussed.
\end{abstract}




\section{Introduction}

A correspondence between the large $N$ limit of certain superconformal theories and supergravity theories on the boundary of Anti-de Sitter spaces has been conjectured in [1]. An AdS/CFT correspondence was used in [2] in context of physics of non-extremal black holes.

An AdS/CFT relationship between correlation functions in the large $N$ CFT and the classical supergravity action on the boundary was proposed in [3, 4] in terms of the component supergravity and in [5] in superspace formulation. The proposal is that the generating functional of the correlation functions in the large $N$ CFT is given by the supergravity action considering as a functional of the boundary values of the fields.

Construction of the $n$-point correlation functions for scalar fields within the proposal [4] has been presented in [6] and it is considered in more detail in [7]. The 3-point amplitudes including vector fields in the AdS supergravity are considered in 99. The free spinor fields are discussed in [8] and amplitudes including graviton are considered in [10]. For other recent considerations of the AdS/CFT correspondence see [12]- 47].

To make the proposal more precise one has to explain the meaning of the boundary action. The boundary action is not well defined for free fields and one gets divergencies when one considers the value of the classical action on the solutions of the corresponding boundary problem. In [6] it was proposed to make a renormalization of the boundary action to get the well defined functional and the breaking of conformal invariance has been pointed out. In [6] only massless scalar field have been considered. In this note we shall discuss the renormalization of the boundary action for the massive scalar fields.

In 23] the breaking of conformal invariance by the irrelevant Born-Infeld corrections to the Yang-Mills theory is considered. These effects go beyond "the strict conformal limit". In this note we discuss the breaking of conformal invariance that takes place already in the strict conformal limit.

Actually the proposals for the AdS/CFT correspondence in [3] and in [4] are somewhat different. In [3] a cut-off on the boundary of AdS is introduced and to get the 2-point correlation function in CFT it is proposed to take only "the leading nonanalytical term" in the Fourier transform of the boundary action. Unfortunately this "non-analytic" prescription depends on the used regularisation procedure and more specifically on the cut-off.

In [4] an elegant expression for the boundary action for free fields has been proposed. In the four dimensional Euclidean space for the massless fields it has the form

$$
I\left(\Phi_{0}\right)=\frac{C}{2} \int \frac{\Phi_{0}(\mathbf{x}) \Phi_{0}(\mathbf{y})}{|\mathbf{x}-\mathbf{y}|^{8}} d \mathbf{x} d \mathbf{y}
$$

Here $\Phi_{0}(\mathbf{x})$ is a test function in the four dimensional space which is considered as the boundary function for a field in the AdS space.

The integral (1) requires a regularisation. If one interprets it as the value of the distribution $|\mathbf{x}|^{-8}$ on the test function then one has the breaking of the conformal symmetry because the distribution $|\mathbf{x}|^{-8}$ is not a homogeneous generalized function, see [6] for a discussion of this point. Let us recall that the function $|\mathbf{x}|^{-8}$ of course is 
scaling invariant for $\mathbf{x} \neq 0$ but in (1) one has the integral and it leads to divergencies if we don't regularise it. The regularisation leads to the breaking of the conformal invariance.

In other words if in CFT one has the correlator of the form

$$
<\mathcal{O}(\mathbf{x}) \mathcal{O}(\mathbf{y})>=\frac{C}{|\mathbf{x}-\mathbf{y}|^{8}}
$$

then it is scaling invariant for $\mathbf{x} \neq \mathbf{y}$ because one can consider it as an ordinary function. However if we want to define the correlation function also for $\mathbf{x}=\mathbf{y}$ in the distribution sense (for example if we want to integrate it as in (11)) then one has the breaking of the conformal symmetry.

In [6] the following renormalized boundary action is obtained (see also the discussion in the next section)

$$
I_{r e n}\left(\Phi_{0}\right)=\int_{R^{4}} d \mathbf{x} \Phi_{0}(\mathbf{x})\left[a \Delta^{2} \log (-\Delta)+b \Delta^{2}+c \Delta\right] \Phi_{0}(\mathbf{x})
$$

where $\Delta$ is the Laplacian and $a, b, c$ are arbitrary constants.

The action (3) is not conformally invariant. If we assume 'the minimal breaking of conformal symmetry" (or a minimal subtraction renormalization scheme [6]) and take $c=0$ then we obtain the renormalized boundary action

$$
I_{r e n}\left(\Phi_{0}\right)=\int_{R^{4}} d \mathbf{x} \Phi_{0}(\mathbf{x})\left[a \Delta^{2} \log (-\Delta)+b \Delta^{2}\right] \Phi_{0}(\mathbf{x})
$$

The renormalized action (4) (or (3)) gives an interpretation of the formula (1) if we treat $|\mathbf{x}-\mathbf{y}|^{-8}$ as a distribution because the Fourier transform of the distribution $|\mathbf{x}|^{-8}$ is $a p^{4} \log p+b p^{4}$.

\section{Massless Fields}

Let us recall first the renormalization of the boundary action for massless fields [6]. The AdS action for this theory in the Lobachevsky space $R_{+}^{d+1}=\left\{\left(x_{0}, \mathbf{x}\right) \in R^{d+1} \mid x_{0}>0\right\}$ is given by

$$
I=\frac{1}{2} \int_{\epsilon}^{\infty} d x_{0} \int_{R^{d}} d \mathbf{x} \frac{1}{x_{0}^{d-1}} \sum_{i=0}^{d}\left(\frac{\partial \Phi}{\partial x_{i}}\right)^{2}
$$

Here $\epsilon>0$ is a cut-off, see [3]. The solution of the Dirichlet problem

$$
\left(\sum_{i=0}^{d} \frac{\partial^{2}}{\partial x_{i}^{2}}-\frac{(d-1)}{x_{0}} \frac{\partial}{\partial x_{0}}\right) \Phi=0,\left.\quad \Phi\right|_{x_{0}=0}=\Phi_{0}(\mathbf{x})
$$

can be represented in the form

$$
\Phi\left(x_{0}, \mathbf{x}\right)=c x_{0}^{d / 2} \int_{R^{d}} d \mathbf{p} e^{i \mathbf{p x}}|\mathbf{p}|^{\frac{d}{2}} K_{\frac{d}{2}}\left(|\mathbf{p}| x_{0}\right) \tilde{\Phi}_{0}(\mathbf{p})
$$


where $K_{\frac{d}{2}}(y)$ is the modified Bessel function. By integrating by parts, one can rewrite (5) as

$$
I=-\left.\frac{1}{2} \int_{R^{d}} d \mathbf{x}\left(\frac{1}{x_{0}^{d-1}} \Phi \frac{\partial \Phi}{\partial x_{0}}\right)\right|_{x_{0}=\epsilon}
$$

Using the asymptotic expansion of the modified Bessel function one gets a regularised expression for the action.

For $d=4$ one has for $x_{0} \rightarrow 0$

$$
\Phi\left(x_{0}, \mathbf{x}\right)=C \int_{R^{4}} d \mathbf{p} e^{i \mathbf{p x}}\left[2-\frac{1}{2}\left(x_{0} p\right)^{2}-\frac{\left(x_{0} p\right)^{4}}{8} \log \frac{x_{0} p}{2}+c\left(x_{0} p\right)^{4}+\ldots\right] \tilde{\Phi}_{0}(\mathbf{p}),
$$

here $p=|\mathbf{p}|$. The action (8) for $\epsilon \rightarrow 0$ behaves as

$$
I=C \int_{R^{4}} d \mathbf{p}\left|\tilde{\Phi}_{0}(\mathbf{p})\right|^{2}\left[-\frac{1}{\epsilon^{2}} p^{2}-\frac{p^{4}}{2} \log \frac{\epsilon p}{2}+c_{1} p^{4}+\ldots\right] .
$$

The appearance of divergent terms in the classical action can be related with the fact that the propagator for a field $\mathcal{O}$ of conformal dimension 4 should be a multiple of $|\mathbf{x}-\mathbf{y}|^{-8}$ and one has to define it as a distribution.

In the spirit of the minimal subtractions scheme in the theory of renormalization one can write a "renormalized" action as

$$
I_{\text {ren }}=\int_{R^{4}} d \mathbf{p}\left|\tilde{\Phi}_{0}(\mathbf{p})\right|^{2}\left[a p^{4} \log p^{2}+b p^{4}\right] .
$$

One can write the final result as follows

$$
I=\int_{R_{+}^{5}} d x \sqrt{g}(\nabla \Phi)^{2} \longrightarrow I_{r e n}=\int_{R^{4}} d \mathbf{x} \Phi_{0}\left[a \Delta^{2} \log (-\Delta)+b \Delta^{2}\right] \Phi_{0}
$$

where the arrow includes the renormalization. The renormalized action includes a local term

$$
\int_{R^{4}} d \mathbf{x}\left(\Delta \Phi_{0}\right)^{2}
$$

There is also a non-local term. This is related with the fact that the distribution $|\mathbf{x}|^{-8}$ [11] is not a homogeneous in $R^{4}$ (there is the logariphm in the scaling law).

If one adds also finite parts, then one gets a term $\Phi_{0} \Delta \Phi_{0}$. One requires additional physical assumptions to fix the form of the renormalized action. There is an analogy with the choice of "the leading non-analitical term" in [3].

One can consider the renormalized action (11) as a definition of distribution $|\mathbf{x}-\mathbf{y}|^{-\mathbf{8}}$ and interpret the action [四]

$$
\int_{R^{2 d}} \frac{\Phi_{0}(\mathbf{x}) \Phi_{0}(\mathbf{y})}{|\mathbf{x}-\mathbf{y}|^{2 d}} d \mathbf{x} d \mathbf{y}
$$

as the value of the distribution $|\mathbf{x}-\mathbf{y}|^{-2 d}$ on a test function. The Fourier transform of this distribution [11] includes a logariphmic term. For $d=4$ one has

$$
\mid \widetilde{\left.\mathbf{x}\right|^{-8}}=a p^{4} \log p+b p^{4},
$$


The distribution $|\mathbf{x}|^{-8}=r^{-8}$ is the so called associated generalized function. It is obtained by taking the analytical continuation over $\lambda$ of the distribution $r^{\lambda}$ and then expanding at a pole. In the $d$-dimensional space one has at $\lambda=-d-2 k$ the expansion

$$
r^{\lambda}=\omega_{d}\left[\frac{\delta^{(2 k)}(r)}{(2 k) !} \frac{1}{\lambda+d+2 k}+r^{-d-2 k}+(\lambda+d+2 k) r^{-d-2 k} \log r+\ldots\right]
$$

where $\omega_{d}=2 \pi^{d / 2} / \Gamma(d / 2)$.

\section{Massive Fields}

Let us discuss now the massive case. The free action in the AdS space has the form

$$
I=\frac{1}{2} \int_{\epsilon}^{\infty} d x_{0} \int_{R^{d}} d \mathbf{x}\left[\frac{1}{x_{0}^{d-1}} \sum_{i=0}^{d}\left(\frac{\partial \phi}{\partial x_{i}}\right)^{2}+\frac{m^{2}}{x_{0}^{d+1}} \phi^{2}\right]
$$

Let us consider a solution of the following boundary problem

$$
\left(\sum_{i=0}^{d} \frac{\partial^{2}}{\partial x_{i}^{2}}-\frac{(d-1)}{x_{0}} \frac{\partial}{\partial x_{0}}-\frac{m^{2}}{x_{0}^{2}}\right) \Phi=0,\left.\quad\left(x_{0}\right)^{k} \Phi\right|_{x_{0}=0}=\Phi_{0}(\mathbf{x})
$$

where $k$ is the larger root of the equation

$$
m^{2}=k(d+k)
$$

This solution can be represented in the form

$$
\Phi\left(x_{0}, \mathbf{x}\right)=c x_{0}^{d / 2} \int_{R^{d}} d \mathbf{p} e^{i \mathbf{p x}}|\mathbf{p}|^{\frac{d}{2}+k} K_{\Delta}\left(|\mathbf{p}| x_{0}\right) \tilde{\Phi}_{0}(\mathbf{p})
$$

where

$$
\Delta=\sqrt{\left(\frac{d}{2}\right)^{2}+m^{2}}
$$

Let us consider the case of integer $k$. For even $d$ by using the expansion for $x_{0} \rightarrow 0$

$$
\begin{gathered}
K_{\frac{d}{2}+k}\left(x_{0} p\right)=\frac{1}{\left(x_{0} p\right)^{\frac{d}{2}+k}}\left[\alpha_{0}+\alpha_{1} x_{0} p+\ldots\right]+ \\
\log \left(x_{0} p\right) \cdot\left(x_{0} p\right)^{\frac{d}{2}+k}\left[\beta_{0}+\beta_{1} x_{0} p+\ldots\right]+\left(x_{0} p\right)^{\frac{d}{2}+k}\left[\gamma_{0}+\gamma_{1} x_{0} p+\ldots\right]
\end{gathered}
$$

we get

$$
\begin{gathered}
\Phi\left(x_{0}, \mathbf{x}\right)=c \int_{R^{d}} d \mathbf{p} e^{i \mathbf{p x}}\left[\frac{1}{\left(x_{0} p\right)^{k}}\left(\alpha_{0}+\alpha_{1} x_{0} p+\ldots\right)\right. \\
+\left(x_{0} p\right)^{d+k} \log \left(x_{0} p\right)\left(\beta_{0}+\beta_{1}\left(x_{0} p+\ldots\right)+x_{0} p\left(\gamma_{0}+\gamma_{1} x_{0} p+\ldots\right)\right] p^{k} \tilde{\Phi}_{0}(\mathbf{p}),
\end{gathered}
$$

and

$$
\left.\left(x_{0}\right)^{k} \Phi\left(x_{0}, \mathbf{x}\right)\right|_{x_{0}=0}=c \alpha_{0} \int_{R^{d}} d \mathbf{p} e^{i \mathbf{p x}} \tilde{\Phi}_{0}(\mathbf{p})
$$


By integrating by parts, one can rewrite (17) as

$$
I=-\left.\frac{1}{2} \int_{R^{d}} d \mathbf{x}\left(\frac{1}{x_{0}^{d-1}} \Phi \frac{\partial \Phi}{\partial x_{0}}\right)\right|_{x_{0}=\epsilon}
$$

Using the asymptotic expansion of the modified Bessel function one gets a regularised expression for the action. By using (23) we obtain that the action (25) for $\epsilon \rightarrow 0$ behaves as

$$
I=\int_{R^{4}} d \mathbf{p}\left|(p)^{2 k} \tilde{\Phi}_{0}(\mathbf{p})\right|^{2}\left[\frac{1}{\epsilon^{d}} \sum_{n=-2 k}^{d}(p \epsilon)^{n} a_{n}+a p^{d} \log (\epsilon p)\right] .
$$

Performing the minimal subtractions as in the massless case we get the renormalized boundary action

$$
I_{\text {ren }}=\int_{R^{d}} d \mathbf{p}\left|\tilde{\Phi}_{0}(\mathbf{p})\right|^{2}\left[a p^{d+2 k} \log p+b p^{d+2 k}\right] .
$$

In principle we have to add to (27) also other terms corresponding to the divergent terms in $(10)$.

One can write the final result as follows

$$
I=\int_{R_{+}^{d+1}} d x \sqrt{g}\left[(\nabla \Phi)^{2}+m^{2}(\Phi)^{2}\right] \longrightarrow I_{r e n}=\int_{R^{d}} d \mathbf{x} \Phi_{0}(-\Delta)^{d / 2+k}[a \log (-\Delta)+b] \Phi_{0}
$$

where the arrow includes the renormalization. If one adds also finite parts, then one gets terms $\Phi_{0}(-\Delta)^{l} \Phi_{0}, l=1, \ldots, d / 2+k-1$. One requires additional physical assumptions to fix the form of the renormalized action. As for massless case the renormalized action includes the local term as well as the non-local term. The origine of this fact is that the distribution $|\mathbf{x}|^{-2 d-2 k}$ 11] is not a homogeneous in $R^{d}$ (there is the logariphm in the scaling law).

One can consider the renormalized action (11) as a definition of distribution $\mid \mathbf{x}-$ $\left.\mathbf{y}\right|^{-\mathbf{2}(\mathbf{d}+\mathbf{k})}$ and interpret the action $[4$

$$
\int_{R^{2 d}} \frac{\Phi_{0}(\mathbf{x}) \Phi_{0}(\mathbf{y})}{|\mathbf{x}-\mathbf{y}|^{2 d+2 k}} d \mathbf{x} d \mathbf{y}
$$

as the value of the distribution $|\mathbf{x}-\mathbf{y}|^{-2 d-2 k}$ on a test function. The Fourier transform of this distribution [11] includes a logariphmic term. For $d$ even one has

$$
|\mathbf{x}|^{-2(d+k)}=a p^{d+2 k} \log p+b p^{d+2 k}
$$

\section{Conclusion}

In this note the renormalized boundary action (27), 28) for the free massive scalar field is obtained. There is the breaking of the conformal symmetry in the AdS/CFT correspondence of the same type as it was found earlier for the massless case [6]. 
In this note only the simplest examples of the AdS/CFT correspondence have been discussed. It is interesting to study how the consideration of interaction and the $n$ point correlation functions ( let us recall that in the standard background formalism 48 the behaviour of the two-point function is more subtle then the $n$-point functions) and also supersymmetry will affect the breaking of conformal symmetry.

The work is supported in part by INTAS grant 96-0698. I.A. is supported also by RFFI grant 96-01-00608 and I.V. is supported in part by RFFI grant 96-01-00312.

\section{References}

[1] J. Maldacena, The large N limit of superconformal field theories and supergravity, hepth/9711200

[2] K. Sfetsos and K. Skenderis, Microscopic derivation of the Bekenstein-Hawking entropy formula for non-extremal black holes, hep-th/9711138,

H.J. Boonstra, B. Peeters and K. Skenderis, hep-th/9801076

[3] S.S. Gubser, I.R. Klebanov and A.M. Polyakov, Gauge theory correlators from noncritical string theory, hep-th/9802109

[4] E.Witten, The large N limit of superconformal field theories and supergravity, hepth/9802150

[5] S.Ferrara, C. Fronsdal and A. Zaffaroni, On N=8 Supergravity On Ads(5) And N=4 Superconformal Yang-Mills Theory, hep-th/9802203.

[6] I.Ya. Aref'eva and I.V. Volovich, On Large N Conformal Theories, Field Theories In Anti-De Sitter Space And Singletons, hep-th/9803028

[7] W. Mück and K.S. Viswanathan, Conformal field theory correlators from classical scalar field theory on $\mathrm{AdS}_{d+1}$, hep-th/9804035.

[8] M.Henningson and K.Sfetsos, Spinors and the AdS/CFT correspondence, hepth/9803251.

[9] D.Z. Freedman, S.D. Mathur, A. Matusis and L. Rastelli, Correlation functions in the $\mathrm{CFT}_{d} / \mathrm{AdS}_{d+1}$ correspondence, hep-th/9804058.

[10] Hong Liu and A.A. Tseytlin, D=4 Super Yang-Mills, D=5 gauged supergravity, and $D=4$ conformal supergravity, hep-th/9804083

[11] I.M.Gelf'and and G.E.Shilov, Generalized Functions, Academic press, 1963

[12] N. Itzhaki, J. M. Maldacena, J. Sonnenschein, and S. Yankielowicz, hep-th/9802042.

[13] M. Gunaydin and D. Minic, Singletons, Doubletons and M-theory, hep-th/9802047

[14] G. T. Horowitz and H. Ooguri, Spectrum of Large N Gauge Theory from Supergravity, hep-th/9802116 
[15] S. Ferrara and C. Fronsdal, Gauge fields as composite boundary excitations, hepth/9802126.

[16] S. Kachru, E. Silverstein, '4-D Conformal Theories And Strings On Orbifolds', hepth/9802183

[17] M. Berkooz, 'A Supergravity Dual Of A $(1,0)$ Field Theory In Six-Dimensions', hepth/9802195

[18] V. Balasubramanian and F. Larsen, hep-th/9802198

[19] S.-J. Rey, J. Yee, 'Macroscopic Strings As Heavy Quarks In Large N Gauge Theory And Anti-De Sitter Supergravity', hep-th/9803001

[20] J. Maldacena, 'Wilson Loops In Large N Field Theories', hep-th/9803002

[21] M. Flato, C. Fronsdal, 'Interacting Singletons', hep-th/9803013

[22] A. Lawrence, N. Nekrasov and C. Vafa, hep-th/9803015

[23] S. Gubser, A. Hashimoto, I.R. Klebanov, M. Krasnitz, 'Scalar Absorption And The Breaking Of The World Volume Conformal Invariance', hep-th/9803023

[24] L. Castellani, A. Ceresole, R. D'Auria, S. Ferrara, P. Fre, M. Trigiante, 'G/H M-Branes And Ads $(\mathrm{P}+2)$ Geometries', hep-th/9803039

[25] Ofer Aharony, Yaron Oz, Zheng Yin, 'M Theory On $A d S_{p} \times S^{11-p}$ and Superconformal Field Theories', hep-th/9803051

[26] S. Minwalla, 'Particles On Ads(4/7) And Primary Operators On M(2)-Brane And M(5)Brane World Volumes', hep-th/9803053

[27] S. Ferrara, A. Zaffaroni, N=1,2 4D Superconformal Field Theories and Supergravity in $A d S_{5}$ hep-th/9803060

[28] R.G. Leigh and M.Rozali, The large N limit of the $(2,0)$ superconformal field theory. hep-th/9803068

[29] M. Bershadsky, Z. Kakushadze, C. Vafa, String Expansion as Large N Expansion of Gauge Theories, hep-th/9803076.

[30] E. Halyo, Supergravity on $A d S_{4 / 7} \times S^{7 / 4}$ and M Branes, hep-th/9803077

[31] A. Rajaraman, Two-Form Fields and the Gauge Theory Description of Black Holes, hep-th/9803082

[32] E. Bergshoeff, K. Behrndt, D-Instantons and asymptotic geometries, hep-th/9803090

[33] S. Ferrara, A. Kehagias, H. Partouche, A. Zaffaroni, Membranes and Fivebranes with Lower Supersymmetry and their AdS Supergravity Duals, hep-th/9803109

[34] J. Gomis, Anti de Sitter Geometry and Strongly Coupled Gauge Theories, hepth/9803119 
[35] E. Witten, Anti-de Sitter Space, Thermal Phase Transition, And Confinement In Gauge Theories, hep-th/9803131

[36] S.J.Rey. S.Theisen and J.T.Yee, Wilson-Polyakov Loop at Finite Temparature in Large N Gauge Theory and Anti-de-Sitter Supergravity, hep-th/9803135.

[37] A. Brandhuber, N.Itzhaki, J. Sonnenschein, S. Yankielowicz, Wilson Loops, Confinement, and Phase Transitions in Large N Gauge Theories from Supergravity, hepth/9803137

[38] M. Gunaydin, Unitary Supermultiplets of $\operatorname{OSp}(1 / 32, \mathrm{R})$ and M-theory, hep-th/9803138

[39] Y. Oz, J.Terning, Orbifolds of $A d S_{5} x S^{5}$ and 4d Conformal Field Theories, hepth/9803167

[40] I.V. Volovich, Large N Gauge Theories and Anti-de Sitter Bag Model, hep-th/9803174

[41] Z. Kakushadze, Gauge Theories from Orientifolds and Large N Limit, hep-th/9803214.

[42] A. Volovich, Near Anti-de Sitter Geometry and Corrections to the Large N Wilson Loop hep-th/9803220

[43] H. Boonstra, B. Peeters, K. Skenderis, Brane intersections, anti-de Sitter spacetimes and dual superconformal theories, hep-th/9803231

[44] S. Ferrara, A. Kehagias, H. Partouche, A. Zaffaroni, $A d S_{6}$ Interpretation of 5d Superconformal Field Theories, hep-th/9804006

[45] J. Maldacena, A. Strominger AdS3 Black Holes and a Stringy Exclusion Principle, hepth/9804085

[46] V. D. Ivashchuk, Composite p-branes on Product of Einstein Spaces, hep-th/9804113

[47] Ulf H. Danielsson, A. P. Polychronakos, Quarks, monopoles and dyons at large N, hepth/9804141

[48] I.Ya. Aref'eva, L.D. Faddeev and A.A. Slavnov, Theor. Math. Phys.,21(1975)165 